\title{
ON THE IRREDUCIBLE COMPONENTS OF AN IDEAL
}

\author{
William Heinzer \\ Department of Mathematics \\ Purdue University \\ W. Lafayette, IN 47907 \\ email: heinzer@math.purdue.edu \\ L. J. Ratliff, Jr. \\ Department of Mathematics \\ University of California \\ Riverside, CA 92521 \\ email: ratliff@ucrmath.ucr.edu \\ Kishor Shah \\ Department of Mathematics \\ Southwest Missouri State University \\ Springfield, MO 65804 \\ email: kis100f@wpgate.smsu.edu
}

\begin{abstract}
Let $I$ be an $M$-primary ideal in a local ring $(R, M)$ and let $\operatorname{irr}(I)$ denote the set of irreducible components of $I$, where an ideal $q$ is an irreducible component of $I$ if $q$ occurs as a factor in some decomposition of $I$ as an irredundant intersection of irreducible ideals. We give several characterizations of the ideals $\operatorname{in} \operatorname{irr}(I)$ and show that if $J$ is an ideal between $I$ and an irreducible component of $I$, then $J$ is the intersection of ideals in $\operatorname{irr}(I)$. We also exhibit examples showing that there may exist irreducible ideals containing $I$ that contain no ideal in $\operatorname{irr}(I)$. Also, we determine necessary and sufficient conditions that the principal ideal $u R[u, t I]$ of the Rees ring $R[u, t I]$ have a unique cover, and apply this to the study of the form ring of $R$ with respect to $I$.
\end{abstract}


1. INTRODUCTION. The following notation is fixed for this paper: $(R, M)$ is a local ring with identity $1 \neq 0$, and $I$ is an open (=M-primary) ideal in $R$. Our terminology is generally the same as that in $[\mathrm{M}],[\mathrm{N}]$, and $[\mathrm{ZS}]$.

Irreducible ideals have interested us ever since we learned that each ideal in a Noetherian ring is a finite intersection of irreducible ideals. This is a classical result of Emmy Noether [No, Satz II, p. 33], and is the first of four different types of decomposition considered by Noether in $[\mathrm{No}] .{ }^{1}$ Noether's work stimulated an important classical paper on irreducible ideals by Wolfgang Gröbner $[\mathrm{Gr}] .{ }^{2}$ However, apparently since 1934 few papers have been devoted to the study of irreducible ideals and the decomposition of ideals as a finite intersection of irreducible ideals. Our purpose here is to begin such a study. (This study was partly suggested by our work in [HRS1], [HRS2], and [HRS3], where we discovered that irreducible ideals are closely related to the maximal embedded components of an ideal.) Our results in the present paper show that irreducible ideals have some interesting and useful (and, perhaps, unexpected) properties.

In Section 2 we give several characterizations of the irreducible components of $I$, and then show that $n_{i r r}(I)+1$ is an upper bound on the number $n(I)$ of ideals in a decomposition of $I$ as an irredundant intersection of irreducible ideals, where $n_{\text {irr }}(I)=\min \{\ell(q / I) ; q$ is an irreducible ideal in $R$ that contains $I\}$.

In Section 3 it is shown in (3.2) that each ideal $J \in \mathbf{I}(I)=\{J ; I \subseteq J \subseteq q$ for some ideal $q \in \operatorname{irr}(I)\}$ is the intersection of the ideals $\operatorname{in} \operatorname{irr}(I)$ that contain $J$. Then we characterize the maximal reducible ideals in $\mathbf{I}(I)$ and also show that the ideals in $\mathbf{I}(I)$ that are minimal with respect to properly containing $I$ are the covers of I. Also, we briefly consider the concept of an ideal $J$ being irreducibly related to $I$ (where $J$ is irreducibly related to $I$ in case $J$ is a finite intersection of ideals in $\operatorname{irr}(I))$.

In Section 4 the ideal structure of the Artinian Gorenstein local ring $R=F[x, y]=$ $F[X, Y] /\left(X^{3}, Y^{3}\right)$ (where $F$ is the field $\left.\{0,1\}\right)$ is considered. The main result shows that the ideal $(x+y) R$ is an irreducible ideal that contains $x^{2} y^{2} R$, but contains no irreducible component of $x^{2} y^{2} R$. A large part of this section is devoted to describing

\footnotetext{
${ }^{1}$ An informative discussion of Noether's work in [No] is presented by Robert Gilmer in [Gi].

${ }^{2}$ Gröbner in his paper thanks E. Noether for direction and valuable advice.
} 
how the computer program Macaulay [BS] was used in developing this example.

The main result in Section 5 gives a useful characterization of when the principal ideal $u R[u, t I]$ has a unique cover, and this is then used to show that if $R$ is an Artinian Gorenstein local ring, then $u R[u, t I]$ is irreducible if and only if $R[u, t I]$ is Gorenstein if and only if the form $\operatorname{ring} \mathbf{F}(R, I)$ of $R$ with respect to $I$ is Gorenstein.

Finally, in Section 6 we give three examples of rather "bad" behavior of irreducible ideals. All three examples are in a regular local $\operatorname{ring} R$ of altitude two. The first shows that the set $\mathbf{S}=\left\{I ; n(I) \leq n_{\text {irr }}(I)\right\}$ is nonempty. The next is an example of an infinite descending chain of open ideals $I_{1} \supset I_{2} \supset \ldots$ in $R$ and an infinite set $\mathbf{Q}$ of irreducible ideals $q_{i}$ in $R$ such that, for all positive integers $n$ and $k, \operatorname{irr}\left(I_{n+k}\right) \cap \mathbf{Q}$ $=\left\{q_{1}, \ldots, q_{n+k}\right\}$ and $I_{n+k}=q_{n} \cap q_{n+k}$. The final example shows that if $k<m$ are positive integers, then there exists an open ideal $I$ in $R$ such that $n(I)=m$ and there exists an ideal $J \in \mathbf{I}(I)$ such that $J$ is the irredundant intersection of $m+k$ ideals in $\operatorname{irr}(I)$.

2. THE IDEALS IN $\operatorname{irr}(\mathbf{I})$. In this section we give several definitions that are needed in what follows, recall several facts concerning irreducible ideals that have previously appeared in the literature, then give several characterizations of the ideals in $\operatorname{irr}(I)$, and then show that $n_{\text {irr }}(I)+1$ is an upper bound on $n(I)$.

We begin with several definitions.

(2.1) DEFINITION. Let $J$ be an ideal in a Noetherian ring $A$. Then:

(2.1.1) $J$ is reducible in case there exist ideals $K$ and $L$ in $A$ that properly contain $J$ such that $J=K \cap L . J$ is irreducible in case $J$ is not reducible. An ideal $q$ is an irreducible component of $J$ in case $q$ appears as a factor in a decomposition of $J$ as an irredundant intersection of irreducible ideals (see (2.2.3)).

(2.1.2) $\operatorname{irr}(J)=\{q ; q$ is an irreducible component of $J\}$, and $\mathbf{I}(J)$ denotes the set of all intersections of the ideals in $\operatorname{irr}(J)$ (excluding $A$, the empty intersection).

(2.1.3) $n(J)$ denotes the number of ideals in a decomposition of $J$ as an irredundant intersection of irreducible ideals (see (2.2.3) and (2.2.4)).

(2.1.4) If $A$ is local, then $n_{i r r}(J)=\min \{\ell(q / J) ; q$ is an irreducible ideal in $A$ that contains $J$, where $\ell(q / J)$ denotes the length of the $A$-module $q / J$. 
(2.1.5) If $A$ is local, then $\mathbf{S}=\left\{J ; n(J) \leq n_{\text {irr }}(J)\right\}$. (The letter $\mathrm{S}$ is an abbreviation for "short" - the ideals $J$ in $\mathbf{S}$ have a shorter irreducible decomposition than $n_{i r r}(J)+$ 1 (see (2.5.3).)

(2.1.6) An ideal $K$ is a cover of $J$ in case $J \subset K$ and there exist no ideals between $J$ and $K$. (In this case, $K / J \cong A / N$ for some maximal ideal $N$ in $A$, and it then follows that $K=(J, b) A$ for some $b \in N$ and $N K \subseteq J$.) Also, $K$ is an irreducible cover of $J$ in case $K$ is an irreducible ideal and a cover of $J$.

Concerning (2.1.4), let $\mathbf{J}=\{q ; q$ is an irreducible ideal in $R$ that contains $J\}$, so $\operatorname{irr}(J) \subseteq \mathbf{J}$, so $n_{\text {irr }}(J) \leq \min \{\ell(q / J) ; q \in \operatorname{irr}(J)\}$. If $J$ is an open ideal, then an interesting question is whether this inequality is always an equality. We show in (4.1) that there may exist ideals that are minimal in $\mathbf{J}$ that are not in $\operatorname{irr}(J)$. This indicates that there is a possibility that $n_{\text {irr }}(J)$ strictly less than $\min \{\ell(q / J) ; q \in$ $\operatorname{irr}(J)\}$ may be achievable in an appropriate example.

A number of known results concerning irreducible ideals will be frequently used below, so we briefly summarize them here.

(2.2) REMARK. Let $I$ be an open ideal in a local ring $(R, M)$. Then:

(2.2.1) [ZS, Theorem 34, p. 248] $I$ is irreducible if and only if $I$ has a unique cover (and then its unique cover is $I: M$ ).

(2.2.2) If $I, J$, and $q$ are open ideals in $R$ such that $I \nsubseteq J$ and $q$ is maximal with respect to containing $I$ and not containing $J$, then $q$ is irreducible.

(2.2.3) [No, Satz II and Satz IV] $I$ is a finite intersection of irreducible ideals, and if the intersection is irredundant, then the number of such ideals is the same for each such representation of $I$.

(2.2.4) $[\operatorname{HRS} 2,(3.3 .3)] n(I)=\operatorname{dim}_{R / M}(S(R / I))$, where $S(R / I)$ is the socle $(0)$ : $(M / I)$ of $R / I$; see [SV, p. 69].)

(2.2.5) [HRS2, (3.2)] If $q \in \operatorname{irr}(I)$ and $J \nsubseteq q$ is an ideal between $I$ and $I: M$, then $(q / I) \cap(J / I)$ is a codimensional one subspace of the $R / M$ vector space $J / I$.

(2.2.6) [HRS3, (3.2)] There are no containment relations among the ideals in $\operatorname{irr}(I)$.

Proof. The proofs of these, except for (2.2.2), are given in the cited references. 
For (2.2.2), it is clear that every ideal that contains $q$ must contain $J$, so $q+J$ is the unique cover of $q$, so (2.2.2) follows from (2.2.1),

In (2.3) we give two useful characterizations of the ideals in $\operatorname{irr}(I)$.

(2.3) THEOREM. Let I be an open ideal in a local ring $(R, M)$. Then the following are equivalent for an ideal $q$ in $R$ :

(2.3.1) $q \in \operatorname{irr}(I)$.

(2.3.2) $q$ is irreducible, $I \subseteq q$, and $I: M \nsubseteq q$.

(2.3.3) $q$ is an ideal that is maximal with respect to: (a) containing some ideal $J$ that contains $I$; and, (b) not containing $I: M$.

Proof. It is shown in [HRS2, (3.4)] that an irreducible ideal $q$ in $R$ is in $\operatorname{irr}(I)$ if and only if $I \subseteq q$ and $I: M \nsubseteq q$, so (2.3.1) $\Leftrightarrow(2.3 .2)$.

If (2.3.2) holds, then $I \subseteq q$, so $I: M \subseteq q: M$ and $q: M$ is the unique cover of $q$, by (2.2.1). Therefore every ideal that properly contains $q$ must contain $I: M$, so $q$ is maximal with respect to $(2.3 .3)$ (a) (with $J=I$ ) and (2.3.3)(b), so (2.3.2) $\Rightarrow$ (2.3.3).

Finally, if (2.3.3) holds, then $q$ is irreducible, by (2.2.2), so since $I \subseteq J \subseteq q$ and $I: M \nsubseteq q,[\operatorname{HRS} 2,(3.4)]$ shows that $q \in \operatorname{irr}(I)$, hence $(2.3 .3) \Rightarrow(2.3 .1)$,

(2.4) COROLLARY. $\operatorname{Irr}(I)=\{q ; q$ is an irreducible ideal in $R, I \subseteq q$, and $q \cap(I: M)$ is covered by $I: M\}=\{q ; q$ is an ideal in $R$ that is maximal with respect to: (a) containing an ideal $J$ that contains $I$; and, (b) intersecting $I: M$ in an ideal that is covered by $I: M\}$.

Proof. This readily follows from (2.3) and (2.2.5),

In (2.5) we note a relation between $n(I)$ and $n_{\text {irr }}(I)$ (see (2.1.3) and (2.1.4)).

(2.5) PROPOSITION (2.5.1). If $I$ is irreducible, then $n(I)=1$ and $n_{\text {irr }}(I)=$ 0 , so $I \notin \mathbf{S}(\operatorname{see}(2.1 .5))$. 
(2.5.2) If $I$ has an irreducible cover $q$ and if $I$ is reducible, then $q \in \operatorname{irr}(I), n(I)=$ 2 , and $n_{\text {irr }}(I)=1$, so $I \notin \mathbf{S}$.

(2.5.3) It is always true that $n(I) \leq n_{\text {irr }}(I)+1$.

Proof. (2.5.1) is clear.

For (2.5.2) assume that $I$ is reducible and that $q$ is an irreducible cover of $I$, and let $Q$ be an ideal in $R$ that is maximal with respect to containing $I$ and not containing $q$. (Such an ideal $Q$ exists, since $I$ is reducible.) Then $Q$ is irreducible, by (2.2.2), and $I=q \cap Q$, hence $q, Q \in \operatorname{irr}(I)$. Therefore it follows that $n(I)=2$ and $n_{\text {irr }}(I)=1=\ell(q / I)$, so $I \notin \mathbf{S}$.

For (2.5.3), let $n_{\text {irr }}(I)=k$, let $q$ be an irreducible ideal in $R$ such that $I \subseteq q$ and $\ell(q / I)=k$, and let $q=q_{0} \supset \cdots \supset q_{k}=I$ be a (maximal) chain of ideals is $R$ of length $k$ between $q$ and $I$. Then, for $i=1, \ldots, k, q_{i-1}$ covers $q_{i}$, so if $q^{(i)}$ is an ideal in $R$ that is maximal with respect to containing $q_{i}$ and not containing $q_{i-1}$, then (2.2.2) shows that $q^{(i)}$ is irreducible, and $q^{(i)} \cap q_{i-1}=q_{i}$. Therefore it follows that $I=q_{0} \cap q^{(1)} \cap \cdots \cap q^{(k)}$, so $n(I) \leq k+1=l_{\text {irr }}(I)+1$,

Note that no irreducible $M$-primary ideal is in the set $\mathbf{S}$ of (2.1.5), by (2.5.1), and a similar statement holds for each reducible $M$-primary ideal that has an irreducible cover, by (2.5.2). So it is natural to wonder if either $\mathbf{S}$ is empty or if the following "converse" of (2.5.2) holds: if $I$ is the irredundant intersection of two irreducible ideals, then $I$ has an irreducible cover. In (6.2.1) and (6.2.2) we give examples of when this converse holds, but (6.2.3) shows that it does not hold in general, so $\mathbf{S}$ is not empty.

3. THE IDEALS IN I(I). The main result in this section, (3.1), shows that if $I \subseteq J$, if $K$ is a cover of $J$, and if $I: M \nsubseteq J$, then there exists $q \in \operatorname{irr}(I)$ such that $q \cap K=J$. An immediate consequence of this is (3.2), which characterizes the ideals in $\mathbf{I}(I)$ by showing that these ideals are precisely the ideals between $I$ and an arbitrary irreducible component of $I$. Then we briefly consider some consequences of (3.2), including the relation of irreducibly related (see (3.6)).

The proof of (3.1) is somewhat similar to the proof of (2.12) in [HRS3]. 
(3.1) THEOREM. Let $J$ be an ideal in $R$ such that $I \subseteq J$ and $I: M \nsubseteq J$, and let $K$ be a cover of $J$. Then there exists $q \in \operatorname{irr}(I)$ such that $q \cap K=J$.

Proof. Note first that if $I$ is irreducible, then $J=I$ (since $I: M \nsubseteq J$ and $I: M$ is the unique cover of $I$ (by $(2.2 .1))$ ), so it follows that $K=I: M$ and we may take $q$ $=I$.

Therefore it may be assumed that $I$ is reducible. Then since $I: M \nsubseteq J$, there exists an ideal $q^{\prime \prime}$ in $R$ that is maximal with respect to containing $J$ and not containing $I: M$. Then $q^{\prime \prime} \in \operatorname{irr}(I)$, by $(2.3 .3) \Rightarrow(2.3 .1)$, and since $K$ covers $J$ it follows that either $q^{\prime \prime} \cap K=J$ (as desired), or $K \subseteq q^{\prime \prime}$.

Therefore it may be assumed that $K \subseteq q^{\prime \prime}$. Then $I: M \nsubseteq K$, since $I: M \nsubseteq q^{\prime \prime}$, so it follows that $J \cap(I: M) \subseteq K \cap(I: M) \subset I: M$. If $J \cap(I: M) \subset K \cap(I: M)$, then let $t \in(K \cap(I: M))-J$. Then if $q^{\prime}$ is an ideal in $R$ that is maximal with respect to containing $J$ and not containing $t$, then it follows that $I: M \nsubseteq q^{\prime}$ (so $q^{\prime}$ $\in \operatorname{irr}(I))$ and $K \nsubseteq q^{\prime}$, so $q^{\prime} \cap K=J$, as desired.

Therefore it may be assumed that $J \cap(I: M)=K \cap(I: M)$. Then since $K$ covers $J$, there exists $x \in K-J$ such that $x M \subseteq J$. Also, since $K \cap(I: M)$ is properly contained in $I: M$, there exists $y \in(I: M)-K$, so let $K^{\prime}=(J, x+y) R$. Then $(x+y) M \subseteq J$, so $K^{\prime}$ covers $J$, and $K^{\prime} \neq K$ (since $x \in K$ and $\left.y \notin K\right)$.

Suppose $J \cap(I: M) \subset K^{\prime} \cap(I: M)$ and let $z \in\left(K^{\prime} \cap(I: M)\right)-J$. Then $z=$ $j+r(x+y)$ for some $j \in J$ and for some $r \in R$. Then $r$ is a unit, since $(x+y) M \subseteq$ $J$ and $z \notin J$. Also, $z-r y \in I: M$ (since $z, y \in I: M)$ and $j+r x \in K$ (since $j \in J$ $\subseteq K$ and $x \in K)$, so $z-r y=j+r x \in K \cap(I: M)=J \cap(I: M)$. Therefore $j+r x$ $\in J$, and $j \in J$ and $r$ is a unit, hence $x \in J$, and this contradicts the choice of $x$.

Therefore it follows that $J \cap(I: M)=K^{\prime} \cap(I: M)$. Therefore let $q$ be an ideal in $R$ that is maximal with respect to containing $K^{\prime}$ and not containing $y$ (where $y$ $\in(I: M)-K)$. Then $I: M \nsubseteq q($ since $y \in(I: M)-q)$, so $q \in \operatorname{irr}(I)$. And $K \nsubseteq$ $q$, since $(J, x+y) R=K^{\prime} \subseteq q$ and $y \notin q$ (so $x \notin q$ and $x \in K$ ). Therefore, since $K$ covers $J$ it follows that $q \cap K=J$,

(3.2) COROLLARY. Assume that $I$ is reducible, let $q \in \operatorname{irr}(I)$, and let $J$ be an ideal in $R$ such that $I \subseteq J \subseteq q$. Then $J \in \mathbf{I}(I)$. In fact, $J$ is the (possibly redundant) 
intersection of $\ell(q / J)+1$ ideals in $\operatorname{irr}(I)$. Therefore $\mathbf{I}(I)=\{J ; J$ is an ideal in $R$ such that $I \subseteq J \subseteq q$ for some $q \in \operatorname{irr}(I)\}=\{J ; J$ is a finite intersection of ideals in $\operatorname{irr}(I)\}$.

Proof. Let $\ell(q / J)=k$ and let $q=q_{0} \supset q_{1} \supset \cdots \supset q_{k}=J$ be a maximal chain of ideals between $q$ and $J$. Then $I: M \nsubseteq q_{0}$, by $(2.3 .1) \Rightarrow(2.3 .2)$, so $I: M \nsubseteq q_{i}$ for $i=1, \ldots, k$, and $q_{i-1}$ covers $q_{i}$, so (3.1) shows that there exists $q^{(i)} \in \operatorname{irr}(I)$ such that $q^{(i)} \cap q_{i-1}=q_{i}$. Therefore it follows that $J=q_{0} \cap q^{(1)} \cdots \cap q^{(k)}$, hence $J$ is the intersection of $\ell(q / J)+1$ ideals $\operatorname{in} \operatorname{irr}(I)$, so $J \in \mathbf{I}(I)$.

Finally, since $R / I$ has finite length, it follows that the ideals in $\mathbf{I}(I)$ are finite intersections of the ideals in $\operatorname{irr}(I)$, so the final statement follows from what was shown in the preceding paragraph,

(3.3) COROLLARY. Assume that $I$ is reducible and let $m=\min (\{\ell(M / q) ; q \in$ $\operatorname{irr}(I)\})$. Then $\operatorname{card}(\{q ; q \in \operatorname{irr}(I)$ and $\ell(M / q)=m\}) \geq 2$.

Proof. Let $q_{1} \in \operatorname{irr}(I)$ such that $\ell\left(M / q_{1}\right)=m$. Then since $\ell\left(q_{1} / I\right)$ is finite, it is clear that there exists an ideal $J$ in $R$ such that $q_{1}$ covers $J$ and $I \subseteq J$. Now $J$ is reducible, by $(2.2 .6)$, so $n(J) \geq 2$. But since $\ell\left(q_{1} / J\right)=1$, (3.2) shows that there exists an ideal $q_{2} \in \operatorname{irr}(I)$ such that $J=q_{1} \cap q_{2}$. Then it follows that $\ell\left(M / q_{2}\right) \leq$ $\ell(M / J)-1=\ell\left(M / q_{1}\right)=m \leq \ell\left(M / q_{2}\right)$ (this last inequality by the definition of $m$ ), hence $\ell\left(M / q_{2}\right)=m=\ell\left(M / q_{1}\right)$,

The next result lists several properties of the ideals in $\mathbf{I}(I)$.

(3.4) PROPOSITION. The following statements hold for an open reducible ideal $I$ :

(3.4.1) The maximal elements in $\mathbf{I}(I)$ are the elements in $\operatorname{irr}(I)$.

(3.4.2) The maximal reducible ideals in $\mathbf{I}(I)$ are the ideals $J \in \mathbf{I}(I)$ such that $n(J)$ $=2$ and $J$ is covered by all ideals $q \in \operatorname{irr}(I)$ that contain $J$.

(3.4.3) $I$ is the minimum element in $\mathbf{I}(I)$.

(3.4.4) The ideals $J$ in $\mathbf{I}(I)$ that are minimal with respect to properly containing $I$ are the ideal covers of $I$, and for each such ideal $J$ it holds that $n(J) \geq n(I)-1$. 
(3.4.5) If $J \in \mathbf{I}(I)$ and if $J=Q_{1} \cap \cdots \cap Q_{h}$ is an arbitrary decomposition of $J$ as an intersection of irreducible ideals, then at least one $Q_{i}$ is in $\operatorname{irr}(I)$.

(3.4.6) If $J \in \mathbf{I}(I)$, if $J=Q_{1} \cap \cdots \cap Q_{h}$ is an arbitrary decomposition of $J$ as an intersection of irreducible ideals, and if $\ell((I: M) /(J \cap(I: M)))=k$, then at least $k$ of the $Q_{i}$ are in $\operatorname{irr}(I)$.

(3.4.7) An ideal $J$ in $R$ is in $\mathbf{I}(I)$ if and only if $I \subseteq J$ and $I: M \nsubseteq J$.

Proof. (3.4.1) is clear by the definitions of $\mathbf{I}(I)$ and $\operatorname{irr}(I)$.

For (3.4.2) let $J \in \mathbf{I}(I)$ such that $n(J)=2$ and $J$ is covered by all ideals $q \in$ $\operatorname{irr}(I)$ such that $J \subseteq q$. To see that $q$ is a maximal reducible ideal in $\mathbf{I}(I)$ let $q^{\prime} \in$ $\mathbf{I}(I)$ such that $q \subset q^{\prime}$ and let $q^{\prime \prime} \in \operatorname{irr}(I)$ such that $q^{\prime} \subseteq q^{\prime \prime}$. Then $q \subset q^{\prime \prime}$, so $\ell\left(q^{\prime \prime} / q\right)$ $=1$, by hypothesis, hence $q^{\prime}=q^{\prime \prime}$, so it follows that $q$ is a maximal reducible ideal in $\mathbf{I}(I)$.

Conversely, let $q$ be a maximal reducible ideal in $\mathbf{I}(I)$. Then $q=q_{1} \cap \cdots \cap q_{k}$ for some ideals $q_{1}, \ldots, q_{k}$ in $\operatorname{irr}(I)$. Assume this intersection is irredundant. Then $k=$ 2, since otherwise $q \subset q_{1} \cap q_{2}$ and $q_{1} \cap q_{2}$ is reducible and is in $\mathbf{I}(I)$. Also, if $q \subset$ $q^{\prime} \in \operatorname{irr}(I)$, and if $\ell\left(q^{\prime} / q\right)>1$, then there exists an ideal $q^{\prime \prime}$ in $R$ such that $q \subset q^{\prime \prime}$ $\subset q^{\prime}$, so $q^{\prime \prime} \in \mathbf{I}(I)$, by (3.2), and $q^{\prime \prime}$ is reducible, by (2.2.6), and this contradicts the choice of $q$. Therefore (3.4.2) holds.

It is clear that $I$ is the minimum element in $\mathbf{I}(I)$, so (3.4.3) holds.

For (3.4.4) let $J$ be a cover of $I$. Then $I \subset J \subseteq I: M$. If $J=I: M$, then since every cover of $I$ is contained in $I: M$ it follows that $I: M$ is the unique cover of $I$, so $I$ is irreducible by (2.2.1), and this contradicts the hypothesis that $I$ is reducible. Therefore $J \subset I: M$, so there exists an ideal $q \in \operatorname{irr}(I)$ such that $J \subseteq q$, by (2.3.3) $\Rightarrow(2.3 .1)$, so $J \in \mathbf{I}(I)$, by (3.2), and it then readily follows that $J$ is minimal in $\mathbf{I}(I)$ with respect to properly containing $I$.

For the converse let $J \in \mathbf{I}(I)$ be minimal with respect to properly containing $I$. Then since every ideal properly between $I$ and $J$ is in $\mathbf{I}(I)$, by (3.2), it follows that $\ell(J / I)=1$, hence $J$ is a cover of $I$. Therefore, if $n(J)=k$ and $J=q_{1} \cap \cdots \cap q_{k}$ is a decomposition of $J$ as an irredundant intersection of irreducible ideals, then (3.1) shows that there exists $q \in \operatorname{irr}(I)$ such that $q_{1} \cap \cdots \cap q_{k} \cap q=I$, hence $n(I) \leq$ $n(J)+1$. 
For (3.4.5), note that $I: M$ is not contained in any ideal in $\operatorname{irr}(I)$, by $(2.3 .1) \Rightarrow$ (2.3.2), so it follows from (3.2) that $I: M \nsubseteq J$. Therefore $I: M \nsubseteq Q_{i}$ for some $i=$ $1, \ldots, h$, and $I \subseteq J \subseteq Q_{i}$, so $Q_{i} \in \operatorname{irr}(I)$ by $(2.3 .2) \Rightarrow(2.3 .1)$.

For (3.4.6) let $j$ such that $Q_{1}, \ldots, Q_{j}$ are in $\operatorname{irr}(I)$ and $Q_{j+1}, \ldots, Q_{h}$ are not in $\operatorname{irr}(I)$, so $j \geq 1$ by (3.4.5). Let $J_{0}=I: M$ and for $i=1, \ldots, j$ let $J_{i}=Q_{i} \cap J_{i-1}$. Then (2.2.5) shows that either $J_{i}$ is a codimensional one subspace of $J_{i-1}$ or $J_{i}=$ $J_{i-1}$. Therefore since $\ell((I: M) /(J \cap(I: M)))=k$ it follows that $j \geq k$.

Finally, for (3.4.7), if $J$ is an ideal in $R$ such that $I \subseteq J$ and $I: M \nsubseteq J$, then (2.3.3) $\Rightarrow(2.3 .1)$ shows that there exists $q \in \operatorname{irr}(I)$ such that $J \subseteq q$, hence $J \in \mathbf{I}(I)$ by $(3.2)$.

And, if $J \in \mathbf{I}(I)$, then $I \subseteq J \subseteq q$ for some $q \in \operatorname{irr}(I)$, by (3.2), and $I: M \nsubseteq q$, by $(2.3 .1) \Rightarrow(2.3 .2)$, hence $I: M \nsubseteq J$,

The next remark generalizes (3.3).

(3.5) REMARK. It follows from (3.4.2) that if $J$ is a maximal reducible ideal in $\mathbf{I}(I)$, then $\ell(M / q)=\ell(M / J)-1$ for all ideals $q \in \operatorname{irr}(I)$ that contain $J$. And there exist at least two such ideals $q$, by (3.2).

We next breifly consider a new relation (called "irreducibly related") between two open ideals in $R$.

(3.6) DEFINITION. If $I$ and $J$ are open ideals in $R$, then it will be said that $J$ is irreducibly related to $I$ in case $J$ is the (finite) intersection of ideals in $\operatorname{irr}(I)$. We will denote this relation by $J \underline{i r} I$.

(3.7) PROPOSITION. The following hold for the relation "irreducibly related":

(3.7.1) The ideals that are irreducibly related to I are the ideals in $\mathbf{I}(I)$.

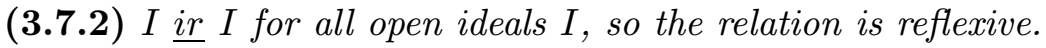

(3.7.3) If $I \underline{i r} J$ and $J \underline{i r} I$, then $I=J$, so the relation is anti-symmetric.

(3.7.4) The relation is not transitive.

(3.7.5) If $I, J$, and $K$ are open ideals in $R$ such that $J \underline{\text { ir }} I, K \underline{i r} J$, and $I: M=$ $J: M$, then $K \underline{i r} I$. 
(3.7.6) If $J$ is an ideal in $R$, then $J \underline{\text { ir }} I$ if and only if $I \subseteq J$ and $I: M \nsubseteq J$.

Proof. (3.7.1) follows immediately from (3.2) and the definitions.

(3.7.2) is clear from the definition, and (3.7.3) follows immediately from (3.7.1).

For (3.7.4) it suffices to give an example of ideals $H, J, K$ such that $J \underline{i r} H$, $K \underline{i r} J$, and $K$ is not irreducibly related to $H$. For this, let $(L, N)$ be a local ring, let $H, J$, and $q$ be open ideals such that $H \subset J \subset q \in \operatorname{irr}(H) \nsupseteq \operatorname{irr}(J)$, and let $K$ $\in \operatorname{irr}(J)-\operatorname{irr}(H)$. Then (3.2) and (3.7.1) show that $J \underline{i r} H, K \underline{i r} J$, and $K$ is not irreducibly related to $H$, hence this relation is not transitive.

For (3.7.5), let $I, J, K$ be open ideals in $R$ such that $J \underline{i r} I, K \underline{i r} J$, and $I: M=$ $J: M$. Then $K=Q_{1} \cap \cdots \cap Q_{h}$ for a finite number of ideals $Q_{1}, \ldots, Q_{h}$ in $\operatorname{irr}(J)$, and for $j=1, \ldots, h$ we have $I \subseteq J \subseteq Q_{j}$ and $I: M=J: M \nsubseteq Q_{j}$, so $Q_{j} \in \operatorname{irr}(I)$, by $(2.3 .2) \Rightarrow(2.3 .1)$. Therefore $K \underline{i r} I$.

Finally, (3.7.6) follows immediately from (3.4.7) and (3.7.1),

\section{MINIMAL IRREDUCIBLES NEED NOT BE IRREDUCIBLE}

COMPONENTS. In this section we consider the ideal structure of the ring $L$ $=F[X, Y] /\left(X^{3}, Y^{3}\right)$ (where $F$ is the field of two elements), and use it to show that it is possible for an irreducible ideal $q$ in a local ring $R$ to contain an open ideal $I$ and yet not contain any irreducible component of $I$. (In this regard, it is shown in [HRS4, Corollary 6.5] that if $I$ is a monomial ideal in a Gorenstein local ring $R$ and if $Q$ is minimal in the set $\{q ; I \subseteq q$ and $q$ is an irreducible monomial ideal in $R\}$, then $Q$ is an irreducible component of $I$. (4.1) shows that the "monomial" condition was crucial for this result.)

(4.1) THEOREM. There exist a local ring $(L, N)$ and open ideals $J \subset q$ such that $q$ is irreducible and contains no irreducible component of $J$.

Proof. (See (4.2.3) for more details concerning the following proof.) Let $F$ be the field of two elements, let $X$ and $Y$ be indeterminates, and let $L=F[X, Y] / K$, where $K=\left(X^{3}, Y^{3}\right) F[X, Y]$, so $L$ is a finite local ring (and $L$ is Gorenstein, since $K$ is irreducible). Let $N=(x, y) L$, where $x=X+K$ and $y=Y+K$, let $J=$ $x^{2} y^{2} L$, and let $q=(x+y) L$. Then it is readily checked that $(0):\left(x^{2}+x y+y^{2}\right) L$ 
$=q$, so $q$ is irreducible (by $\left[\mathrm{ZS}\right.$, Theorem 35 , p.250], since $\left(X^{3}, Y^{3}\right) F[X, Y]_{(X, Y)}$ is irreducible). Also, $J=x y^{2} L \cap x^{2} y L$, so $J$ is reducible. Further, $J=N^{4}, J: N=$ $N^{3}=\left(x^{2} y, x y^{2}\right) L$, and $x^{2} y=x^{3}+x^{2} y=x^{2}(x+y)$ and $x y^{2}=x y^{2}+y^{3}=y^{2}(x+y)$, so $N^{3}=\left(x^{2} y, x y^{2}\right) L \subset q$, hence $q \notin \operatorname{irr}(J)$, by (2.3.2) $\Leftrightarrow(2.3 .1)$. Finally, the only ideals properly between $q$ and $J$ are the ideals $\left(x^{2}+x y, y^{2}+x y\right) L,\left(x^{2}+x y\right) L$, $\left(x y+y^{2}\right) L,\left(x^{2}, y^{2}\right) L, N^{3}, x^{2} y L, x y^{2} L$, and $\left(x^{2} y+x y^{2}\right) L$, and none of these ideals is irreducible by [ZS, Theorem 35, p. 250] (since none of them is the annihilator of a principal ideal; specifically, $\left(x^{2}+x y, y^{2}+x y\right) L=(0):\left(x^{2}+x y+y^{2}, x y^{2}\right) L$, $\left(x^{2}+x y\right) L=(0):\left(x^{2}, x y+y^{2}\right) L,\left(x y+y^{2}\right) L=(0):\left(x^{2}+x y, y^{2}\right) L,\left(x^{2}+y^{2}\right) L=$ (0) : $\left(x^{2}+y^{2}, x y\right) L, N^{3}=(0): N^{2}, x^{2} y L=(0):\left(x, y^{2}\right) L, x y^{2} L=(0):\left(x^{2}, y\right) L$, and $\left.\left.\left(x^{2} y+x y^{2}\right) L=(0):\left(x^{2}, x+y, y^{2}\right) L\right)\right)$,

(4.2) REMARK. (4.2.1) It follows, by passing to $L / J$, that another way to state (4.1) is that there exists an Artinian local ring $(L, N)$ with an irreducible ideal $q$ such that $q$ contains no irreducible component of zero.

(4.2.2) The computation to determine the ideal structure of $L$ in (4.1) was carried out by the computer program Macaulay. This computation also showed that $\left(x^{2}+\right.$ $x+y) L$ is an irreducible ideal that contains $J=x^{2} y^{2} L$ and that does not contain any irreducible component of $J$. (Craig Huneke pointed out to us examples of irreducible ideals $q$ containing $I$ that fail to contain an irreducible component of $I$. One of his examples is in a regular local ring $(R, M)$ of altitude three with $M=$ $(x, y, z) R$ and $I=\left(x^{3}, y^{3}, z^{3}, x y z\right) R$. He argues that with $K=\left(x^{3}, y^{3}, z^{3}\right) R$ and $f$ $=y x^{2}+z y^{2}+x z^{2}$, it follows that $K: f R$ is irreducible, contains $I$, and fails to contain an irreducible component of $I$. His other example is the one presented in (4.1).)

(4.2.3) If $F$ is the field with two elements, then there are $256=2^{8}=(1+1)^{8}=$ $\sum_{i=0}^{8}\left(\begin{array}{l}8 \\ i\end{array}\right)$ nonunits in the ring $L=F[X, Y] /\left(X^{3}, Y^{3}\right)$ (since there are the 8 monomials $\left.x, y, x^{2}, x y, y^{2}, x^{2} y, x y^{2}, x^{2} y^{2}\right)$ ranging from $0, x, \ldots, x^{2} y^{2}, x+y, \ldots, x y^{2}+$ $\left.x^{2} y^{2}, x+y+x^{2}, \ldots, x+y+x^{2}+x y+y^{2}+x^{2} y+x y^{2}+x^{2} y^{2}\right)$, so there are also 256 units (each being of the form $f+1$, where $f$ is a nonunit). It is straightforward to write a computer program to compute and store the nonunits in one file and 
the units in another file. Then to get a list of generators of the distinct principal ideals, Macaulay can quickly compute all unit multiples of each of the nonunits, and this shows that each of the 255 nonzero nonunits is a unit multiple of one of the following 20 polynomials (14 homogeneous, 4 homogenizable (by adjusting weights), and 2 nonhomogenizable): $x, y, x^{2}, x y, y^{2}, x^{2} y, x y^{2}, x^{2} y^{2}, x+y, x^{2}+y, x+y^{2}$, $x^{2}+x+y, x^{2}+x y, x y+y^{2}, x^{2}+y^{2}, x^{2}+x y^{2}, x^{2} y+y^{2}, x^{2}+x y+y^{2}, x^{2} y+x^{2}+x y+y^{2}$, $x^{2} y+x y^{2}$. (There is some symmetry in the generators of the two nonhomogenizable principal ideals; for example, $\left(x^{2}+x+y\right) L=\left(y^{2}+x+y\right) L=(x y+x+y) L$, and $\left(x^{2} y+x^{2}+x y+y^{2}\right) L=\left(x y^{2}+x^{2}+x y+y^{2}\right) L=\left(x^{2} y^{2}+x^{2} y+x^{2}+x y+y^{2}\right) L=$ $\left(x^{2} y^{2}+x y^{2}+x^{2}+x y+y^{2}\right) L$.) [To compute these unit multiples, we created a file ("xy", say) to be fed into Macaulay with the "Macaulay < xy" command. It's first few lines specified: (a) an output file (with Macaulay's "monitor" command); (b) the base ring $A$ (with Macaulay's "ring" command (specifying characteristic 2 and 2 variables)); (c) the kernel $K$ (with Macaulay's "ideal" command (specifying the two generators $\left.x^{3}, y^{3}\right)$ ); and, (d) the factor ring $L=A / K$ (with Macaulay's "qring" command). The next line uses Macaulay's "poly" command (to specify the nonunit polynomial $f$ whose unit multiples are desired), and this is followed by 255 pairs of lines "poly $h\{f\} *(g+1)$ " "type $h$ " (with $g$ varying over the 255 nonzero nonunit polynomials), to compute and display (in the output file) the 255 unit multiples $h=$ $f(g+1)$ of $f)$, and then the file is ended with Macaulay's "exit" command (to exit from Macaulay). By successively changing the definition of $f$ in this file, and then feeding it into Macaulay, it is readily checked that each of the 255 nonzero nonunit polynomials is a unit multiple of one of the 20 polynomials listed above; in this regard, it is useful to write a short program to keep track of the distinct polynomials that are unit multiples of these 20 polynomials, since Macaulay's output from each run on the file "xy" contains 255 polynomials, with at most 32 distinct ones. Using this process, Macaulay shows that there are: 32 unit multiples of each of $x, y, x+y$, $x+y^{2}, x^{2}+y, x^{2}+x+y ; 8$ unit multiples of each of $x^{2}+x y, x y+y^{2}, x y, x^{2}+y^{2} ; 4$ unit multiples of each of $x^{2}, y^{2}, x^{2}+x y^{2}, x^{2} y+y^{2}, x^{2}+x y+y^{2}, x^{2} y+x^{2}+x y+y^{2}$; 2 unit multiples of each of $x^{2} y, x y^{2}, x^{2} y+x y^{2}$; and, 1 unit multiple of $x^{2} y^{2}$.]

Now, since $\left(X^{3}, Y^{3}\right)$ is irreducible in $F\left[X^{3}, Y^{3}\right]_{(X, Y)},[\mathrm{ZS}$, Theorem 35, p. 250] 
shows that an ideal $J$ in $L$ is irreducible if and only if (0): $J$ is a principal ideal. Also, $f L=(0):((0): f L)$, so it follows that there are exactly 20 nonzero irreducible ideals in $L$, namely the 20 nonzero ideals $(0): f L$. Using Macaulay's "quotient" command to compute $(0): f L$, it turns out that the 20 nonzero irreducible ideals are: $N=$ $(x, y) L=(0): x^{2} y^{2} L ;\left(x, y^{2}\right) L=(0): x^{2} y L ;\left(x^{2}, y\right) L=(0): x y^{2} L ;\left(x^{2}, x+y, y^{2}\right) L$ $=(0):\left(x^{2} y+x y^{2}\right) L ; x L=(0): x^{2} L ; x^{2} L=(0): x L ; y L=(0): y^{2} L ; y^{2} L=$ (0) : $y L ;(x+y) L=(0):\left(x^{2}+x y+y^{2}\right) L ;\left(x^{2}+x y+y^{2}\right) L=(0):(x+y) L ;\left(x+y^{2}\right) L$ $=(0):\left(x^{2}+x y^{2}\right) L ;\left(x^{2}+x y^{2}\right) L=(0):\left(x+y^{2}\right) L ;\left(x^{2}+y\right) L=(0):\left(x^{2} y+y^{2}\right) L ;$ $\left(x^{2} y+y^{2}\right) L=(0):\left(x^{2}+y\right) L ;\left(x^{2}+x+y\right) L=(0):\left(x^{2} y+x^{2}+x y+y^{2}\right) L ;$ $\left(x^{2} y+x^{2}+x y+y^{2}\right) L=(0):\left(x^{2}+x+y\right) L ;\left(x^{2}, x y+y^{2}\right) L=(0):\left(x^{2}+x y\right) L ;$ $\left(x^{2}+x y, y^{2}\right) L=(0):\left(x y+y^{2}\right) L ;\left(x^{2}+y^{2}, x y\right) L=(0):\left(x^{2}+y^{2}\right) L ;\left(x^{2}, y^{2}\right) L=$ $(0)$ : xyL. (Macaulay's "quotient" command readily computes $(0): f L$ for the 18 homogenizable polynomials $f$, and $(0): f L$ can be computed for the two remaining polynomials $f=x^{2}+x+y$ and $f=x^{2} y+x^{2}+x y+y^{2}$ by having Macaulay compute all the nonunit multiples of each of these two polynomials $f$.)

This has introduced eight new (non-principal) ideals (namely, $N,\left(x, y^{2}\right) L,\left(x^{2}, y\right) L$, $\left(x^{2}, x+y, y^{2}\right) L=\left(x^{2}, x+y\right) L\left(\right.$ since $\left.y^{2}=x^{2}+(x+y)^{2}\right),\left(x^{2}, x y+y^{2}\right) L,\left(x^{2}+x y, y^{2}\right) L$, $\left(x^{2}+y^{2}, x y\right) L$, and $\left(x^{2}, y^{2}\right) L$, and it is straightforward to find eight additional nonprincipal ideals $\left(x^{2}, x y\right) L,\left(x y, y^{2}\right) L,\left(x^{2}, x y^{2}\right) L,\left(x^{2} y, y^{2}\right) L, N^{2}, N^{3}=\left(x^{2} y, x y^{2}\right) L$, $\left(x^{2}+x y, y^{2}+x y\right) L$, and $\left(x^{2}+x y+y^{2}, x y^{2}\right) L ;$ note that these 16 non-principal ideals are all homogeneous. Now Macaulay can be used to show that there are no additional homogeneous ideals (by having it compute a standard basis for each homogeneous ideal obtained by adjoining a homogeneous element to the 30 homogeneous ideals listed above), and then by hand checking it can be seen (by adjoining a nonhomogeneous element to each of these 36 ideals) that there are no additional nonzero proper ideals in $L$.

Next, to determine which irreducible ideals $q$ are in $\operatorname{irr}(J)$ for various ideals $J$, recall that $q \in \operatorname{irr}(J)$ if and only $J \subseteq q$ and $J: N \nsubseteq q$ (by (2.3.2) $\Leftrightarrow(2.3 .1)$ ). With this in mind, Macaulay's "quotient" command can be used to compute $J: N$ for the 34 homogenizable ideals, and then $J: N$ can be computed for the two remaining nonhomogenizable (principal) ideals by computing all nonunit multiples of $x$ and $y$ 
(since $f \in g L: N$ if and only if $f x, f y \in g L$ ).

Finally, the containment relations between these 36 nonzero proper ideals can be determined by having Macaulay compute nonunit multiples of the generators of the ideals.

This procedure is not readily extendible to $F[X, Y] /\left(X^{m}, Y^{n}\right)$ with $m$ and $n$ much larger than 3 ; for example, if $m=3$ and $n=4$, then there are $2^{11}$ nonunit polynomials and 40 nonzero proper principal ideals, and if $m=4=n$, then there are $2^{15}$ nonunit polynomials.

5. WHEN DOES uR[u,tI] HAVE A UNIQUE COVER? In this section we answer this question, and then use it to characterize when $u R[u, t I]$ is irreducible, in the case when $R$ is Artinian. (Here, $R[u, t I]$ is the Rees ring of $R$ with respect to $I$, so $t$ is an indeterminate and $u=1 / t$.)

(5.1) PROPOSITION. Let $\mathbf{R}=R[u, t I]$ and let $\mathbf{M}=(u, M, t I) \mathbf{R}$. Then the following are equivalent:

(5.1.1) $\mathbf{M}$ is a prime divisor of $u \mathbf{R}$.

(5.1.2) There exists a nonnegative integer $k$ and an element $b \in I^{k}$ such that $u \mathbf{R}$ : $b t^{k} \mathbf{R}=\mathbf{M}$.

(5.1.3) There exists a nonnegative integer $k$ and an element $b \in I^{k}$ such that $\left(u, b t^{k}\right) \mathbf{R}$ covers $u \mathbf{R}$.

(5.1.4) There exists a nonnegative integer $k$ and an element $b \in T_{k}-I^{k+1}$, where $T_{k}=I^{k} \cap\left(I^{k+1}: M\right) \cap\left(I^{k+2}: I\right)$.

Proof. Since $u \mathbf{R}$ and $\mathbf{M}$ are homogeneous, the definition of prime divisor shows that (5.1.1) and (5.1.2) are equivalent.

Also, it readily follows from the definition of an ideal cover that (5.1.2) and (5.1.3) are equivalent.

Now assume that (5.1.2) holds. Then it follows that $b \in I^{k}-I^{k+1}$, that $b t^{k} M \subseteq$ $u \mathbf{R}$, and that $b t^{k}(t I) \subseteq u \mathbf{R}$. Therefore $b \in I^{k}-I^{k+1}, b M \subseteq u^{k+1} \mathbf{R} \cap R=I^{k+1}$, and $b I \subseteq u^{k+2} \mathbf{R} \cap R=I^{k+2}$, so it follows that (5.1.4) holds, hence (5.1.2) $\Rightarrow$ (5.1.4).

Finally, if (5.1.4) holds, then $b t^{k} \in \mathbf{R}-u \mathbf{R}, b M \subset I^{k+1}$, and $b I \subseteq I^{k+2}$, so it follows that $b t^{k} M \subseteq t^{k} I^{k+1}=u(t I)^{k+1} \subseteq u \mathbf{R}$, and $b t^{k}(t I) \subseteq t^{k+1} I^{k+2}=u(t I)^{k+2}$ 
$\subseteq u \mathbf{R}$. Therefore it follows that $b t^{k} \in u \mathbf{R}: \mathbf{M}-u \mathbf{R}$, so $u \mathbf{R}: b t^{k} \mathbf{R}=\mathbf{M}$, hence $(5.1 .4) \Rightarrow(5.1 .2)$

(5.2) REMARK. Let $J$ be an ideal in a local ring $(R, M)$ and for each nonnegative integer $k$ let $T_{k}=J^{k} \cap\left(J^{k+1}: M\right) \cap\left(J^{k+2}: J\right)$ (as in (5.1.4)). Then:

(5.2.1) $J^{k+1} \subseteq T_{k}$ for all $k \geq 0$.

(5.2.2) If $J^{k+1} \subset T_{k}$, then $M \in \operatorname{Ass}\left(R / J^{k+1}\right)$.

(5.2.3) If $J$ is regular, then $J^{k+2}: J=J^{k+1}$ for all large $k$, so $T_{k}=J^{k+1}$ for all large $k$.

(5.2.4) If $J=b R$ is a regular principal ideal, then $T_{k}=b^{k+1} R=J^{k+1}$ for all $k \geq$ 0 .

(5.2.5) If $J$ is $M$-primary and $J^{k+1}$ is irreducible, then $J^{k+1}: M$ is its unique cover, so $J^{k+1}: M \subseteq J^{k} \cap\left(J^{k+2}: J\right)$, hence $T_{k}=J^{k+1}: M$ is principal modulo $J^{k+1}$.

Proof. (5.2.1) is clear by the definition of $T_{k}$.

For (5.2.2), if $J^{k+1} \subset T_{k}$, then $J^{k+1} \subset J^{k+1}: M$, so $M \in A s s\left(R / J^{k+1}\right)$.

For (5.2.3), if $J$ is regular, then it is shown in the proof of [RR, (2.1)] that $J^{k+2}: J$ $=J^{k+1}$ for all large $k$, so the definition of $T_{k}$ and (5.2.1) show that $J^{k+1}=T_{k}$ for all large $k$.

For (5.2.4), if $J=b R$ is regular, then it is clear that $J^{k+2}: J=J^{k+1}$ for all $k \geq$ 0 , and it readily follows from this and (5.2.1) that $T_{k}=J^{k+1}$.

Finally, for (5.2.5), if $J$ is $M$-primary and $J^{k+1}$ is irreducible, then $J^{k+1}: M$ is its unique cover, by $(2.2 .1)$, so it follows that $T_{k}=J^{k+1}: M$ is principal modulo $J^{k+1}$

The next result shows an interesting application of the ideals $T_{k}$. (If altitude $(R)$ $=0$, then (5.3) characterizes the ideals in $R$ such that $u \mathbf{R}$ is irreducible; see (5.4).)

(5.3) THEOREM. Let $J$ be an ideal in $R$ and let $\mathbf{R}=R[u, t J]$. Then the following are equivalent:

(5.3.1) $u \mathbf{R}$ has a unique cover.

(5.3.2) There exists a unique nonnegative integer $k$ such that $T_{k} \neq J^{k+1}$, and for this $k, T_{k}$ is a principal ideal modulo $J^{k+1}$, where $T_{k}=J^{k} \cap\left(J^{k+1}: M\right) \cap\left(J^{k+2}: J\right)$. 
Proof. Assume first that (5.3.1) holds and let $f \in \mathbf{R}$ such that $(u, f) \mathbf{R}$ is the unique cover of $u \mathbf{R}$. Then $f \notin u \mathbf{R}$ and $f N \subseteq u \mathbf{R}$ for some maximal ideal $N$ in $\mathbf{R}$, hence $u \mathbf{R}: f \mathbf{R}=N$. Since $u \mathbf{R}$ is homogeneous, it follows that $N=\mathbf{M}=(u, M, t J) \mathbf{R}$. Therefore $\mathbf{M}$ is a prime divisor of $u \mathbf{R}$, so (5.1.1) $\Rightarrow$ (5.1.3) shows that there exists a nonnegative integer $k$ and an element $b \in J^{k}$ such that $\left(u, b t^{k}\right) \mathbf{R}$ is a cover of $u \mathbf{R}$. Therefore the hypothesis implies that $(u, f) \mathbf{R}=\left(u, b t^{k}\right) \mathbf{R}$, so it may be assumed to begin with that $f=b t^{k}$ is homogeneous.

Now $(5.1 .3) \Rightarrow(5.1 .4)$ shows that $b \in T_{k}-J^{k+1}$, so $T_{k} \neq J^{k+1}$. To see that $T_{k}$ modulo $J^{k+1}$ is principal, let $c \in T_{k}-J^{k+1}$. Then (5.1.4) $\Rightarrow$ (5.1.3) shows that $\left(u, c t^{k}\right) \mathbf{R}$ covers $u \mathbf{R}$, so the hypothesis implies that $\left(u, c t^{k}\right) \mathbf{R}=\left(u, b t^{k}\right) \mathbf{R}$, and it then readily follows that $c=x+v b$ for some $x \in J^{k+1}$ and unit $v$ in $R$. Therefore $T_{k}$ is a principal ideal modulo $J^{k+1}$.

Now let $h \neq k$ be a nonnegative integer and suppose there exists $d \in T_{h}-$ $J^{h+1}$. Then $(5.1 .4) \Rightarrow(5.1 .3)$ shows that $\left(u, d t^{h}\right) \mathbf{R}$ is a cover of $u \mathbf{R}$, so $\left(u, d t^{h}\right) \mathbf{R}$ $=\left(u, b t^{k}\right) \mathbf{R}$. If $h<k$, then $d t^{h}=u\left(x t^{h+1}\right)+\left(y u^{h-k}\right)\left(b t^{k}\right)$ for some $x \in J^{h+1}$ and $y \in R$, so by cancelling $t^{h}$ it follows that $d \in J^{h+1}$, and this contradicts the choice of $d$. And a similar computation produces the contradiction that $b \in J^{k+1}$ if $h$ $>k$. Therefore it follows that $h=k$, and this contradicts the choice of $h$, so the supposition that $T_{h}$ properly contains $J^{h+1}$ leads to a contradiction. Therefore $T_{h}$ $=J^{h+1}$ for all nonnegative integers $h \neq k$, hence $(5.3 .1) \Rightarrow(5.3 .2)$.

Now assume that (5.3.2) holds and let $b \in T_{k}-J^{k+1}$. Then (5.1.4) $\Rightarrow(5.1 .3)$ shows that $\left(u, b t^{k}\right) \mathbf{R}$ is a cover of $u \mathbf{R}$. Therefore let $(u, f) \mathbf{R}$ be another cover of $u \mathbf{R}$, so $f \mathbf{M} \subseteq u \mathbf{R}$ and $\mathbf{M}$ and $u \mathbf{R}$ are homogeneous, so it follows that it may be assumed that $f$ is homogeneous, say $f=c t^{h}$. Then (5.1.3) $\Rightarrow(5.1 .4)$ shows that $c$ $\in T_{h}-J^{h+1}$, so (5.3.2) shows that $h=k$ and that $c=x+v b$ for some $x \in J^{k+1}$ and unit $v$ in $R$, so it follows that $\left(u, c t^{h}\right) \mathbf{R}=\left(u, b t^{k}\right) \mathbf{R}$, hence (5.3.2) $\Rightarrow$ (5.3.1),

(5.4) COROLLARY. The following statements are equivalent for an ideal I in an Artinian local ring $(R, M)$ :

(5.4.1) $u \mathbf{R}$ is irreducible.

(5.4.2) (5.3.2) holds. 
(5.4.3) $\mathbf{R}$ is Gorenstein.

(5.4.4) The form ring $\mathbf{F}(R, I)$ of $R$ with respect to $I$ is Gorenstein.

Proof. Since altitude $(R)=0$, it follows that altitude $(\mathbf{R})=1$, so since $u \mathbf{R}$ is homogeneous it follows that $u \mathbf{R}$ is primary for $\mathbf{M}=(u, M, t I) \mathbf{R}$. Therefore $u \mathbf{R}$ is irreducible if and only if $u \mathbf{R}$ has a unique cover (by (2.2.1)), so (5.4.1) $\Leftrightarrow(5.4 .2)$ by $(5.3 .1) \Leftrightarrow(5.3 .2)$.

It is known that $\mathbf{R}$ is Gorenstein if and only if $\mathbf{R}_{\mathbf{M}}$ is Gorenstein [BH, Prop. 3.1.19, page 94 and Ex. 3.6.20, page 142]. Since altitude $\left(\mathbf{R}_{\mathbf{M}}\right)=1$, it follows that $\mathbf{R}_{\mathbf{M}}$ is Gorenstein if and only if $u \mathbf{R}_{\mathbf{M}}$ is irreducible, and $u \mathbf{R}_{\mathbf{M}}$ is irreducible if and only if $u \mathbf{R}$ is irreducible (since $\mathbf{M}$ is the only prime divisor of $u \mathbf{R}$ ), so (5.4.1) $\Leftrightarrow$ (5.4.3).

Finally, $\mathbf{F}(R, I)=\mathbf{R}(R, I) / u \mathbf{R}(R, I)$, so since $u \mathbf{R}$ is regular it follows that (5.4.3) $\Leftrightarrow(5.4 .4)$

(5.5) REMARK. (5.5.1) If $I$ is an ideal in an Artinian local ring $(R, M)$, then $\mathbf{R}$ $=R[u, t I]$ is Cohen-Macaulay. Also, if the equivalent statements of (5.4) hold, then $R$ is Gorenstein.

(5.5.2) If $L=F[X, Y] /\left(X^{3}, Y^{3}\right)$ with $F$ the field with two elements, then the computer program Macaulay can be used to show (by comparing the ideals $T_{k}$ and $I^{k+1}$ for $\left.k=0,1,2,3,4\right)$ that, of the 34 homogenizable ideals in $L$, only the following three choices for $I$ yield that $u L[u, t I]$ is irreducible: $I=x L ; I=y L$; and $I=(x, y) L$. For the ideal $I=x L, T_{k}=I^{k+1}=x^{k+1} L$ for $k \neq 2\left(\right.$ and $x^{k+1} L=$ (0) for $k \geq 2$ ), and $T_{2}=\left(I^{3}, x^{2} y^{2}\right) L=x^{2} y^{2} L$. (Similar results hold for $I=y L$.) And for $I=(x, y) L, T_{k}=I^{k+1}$ for $k \neq 4$, and $T_{4}=x^{2} y^{2} L$ and $I^{5}=(0)$. (It should be noted that all three of these ideals are irreducible, and for the first two of these ideals $I, I^{k}$ is irreducible for all $k \geq 1$.)

(5.5.3) Assume that altitude $(R)>0$, let $J$ be an ideal in $R$, and let $\mathbf{R}=R[u, t J]$. Then neither of the following statements implies the other: (1) $u \mathbf{R}$ has a unique cover. (2) $u \mathbf{R}$ is irreducible.

(5.5.4) If altitude $(R)>0$ and $u \mathbf{R}$ has a unique cover, then $u \mathbf{R}$ is not irreducible (since it is not even primary). 
(5.5.5) If $J$ is a regular principal ideal in $R$, then $u R[u, t J]$ does not have a unique cover.

Proof. For (5.5.1), $u$ is a regular element in $\mathbf{R}=R[u, t I]$ and $\mathbf{M}=(u, M, t I) \mathbf{R}$ has height one, so $\mathbf{R}_{\mathbf{M}}$ is Cohen-Macaulay, so [HR, (4.11)] shows that $\mathbf{R}$ is CohenMacaulay. Also, if (5.4.3) holds, that is, if $\mathbf{R}$ is Gorenstein, then its quotient ring $R[u, t]=\mathbf{R}[1 / u]$ is Gorenstein, so since $t$ is an indeterminate and $u=1 / t$ it follows that $R$ is Gorenstein.

For (5.5.3), if $(R, M)$ is a regular local ring that is not a field and $J=M$, then $u \mathbf{R}$ is prime, and it is clear that $u \mathbf{R}$ has no cover, so (2) does not imply (1). To see that (1) does not imply (2), let $F$ be a field and let $R=F[[X, Y]] /\left(X^{2}, X Y\right)=F[[x, y]]$ and let $J=(x, y) R$. Then the form ring of $R$ with respect to $J$ is $R[u, t J] /(u)$ and is isomorphic to the graded $\operatorname{ring} F[X, Y] /\left(X^{2}, X Y\right)=F[x, y]$, where $x^{2}=x y=0$. Since the ideal (0) of this ring is reducible and has the unique cover $x F[x, y], u \mathbf{R}$ is reducible and has the unique cover $(u, t x) \mathbf{R}$.

For (5.5.4), if altitude $(R)>0$ and $u \mathbf{R}$ has a unique cover, then $u \mathbf{R}$ cannot be irreducible, since $(u, M, t J) \mathbf{R}$ is a prime divisor of $u \mathbf{R}$ (by (5.1.3) $\Rightarrow(5.1 .1))$ and height $((u, M, t J) \mathbf{R})>1$ (so $u \mathbf{R}$ is not even primary).

Finally, for (5.5.5), it follows from (5.2.4) and (5.3) that $u R[u, t J]$ does not have a unique cover,

In passing, it should be noted that (5.5.2) and (5.4.1) $\Rightarrow$ (5.4.4) show that the form $\operatorname{ring} \mathbf{F}=\mathbf{F}(L,(x, y) L)$ is Gorenstein. Another way to see this is to note that $\mathbf{F}$ $=F[X, Y, u, t X, t Y] /\left(u, t^{3} X^{3}, t^{3} Y^{3}\right)$, and $u, t^{3} X^{3}, t^{3} Y^{3}$ is a regular sequence in the locally regular $\operatorname{ring} F[X, Y, u, t X, t Y]$,

(5.6) COROLLARY. If I is an ideal in an Artinian Gorenstein local ring $(R, M)$ such that $u R[u, t I]$ is irreducible, then the integer $k$ such that $T_{k}$ properly contains $I^{k+1}$ is the largest integer $h$ such that $I^{h} \neq 0$, and in this case $T_{k}=b R$, where $b R$ $=(0): M$ is the unique cover of zero in $R$.

Proof. By (5.4.1) $\Rightarrow$ (5.4.2) let $k$ be the integer such that $T_{k}$ properly contains $I^{k+1}$. Choose $h$ such that $I^{h} \neq(0)$ and $I^{h+1}=(0)$, so $T_{h+i}=I^{h+i+1}=(0)$ for all $i \geq 1$, so $k \leq h$. 
Suppose that $k<h$, so there exists $c \in I^{h}$ such that $c \neq 0$. By (5.1.4) $\Rightarrow$ (5.1.3) let $b \in T_{k}-I^{k+1}$ such that $\left(u, b t^{k}\right) \mathbf{R}$ is the unique cover of $u \mathbf{R}$. Then $\left(u, b t^{k}\right) \mathbf{R} \subseteq\left(u, c t^{h}\right) \mathbf{R}$, so $b \in I^{k+1}+c R \subseteq I^{k+1}+I^{h}=I^{k+1}$, and this contradicts the choice of $b$. Therefore it follows that $h=k$, so $I^{k} \neq(0)$ and $I^{k+1}=(0) \subset T_{k}=$ $I^{k} \cap((0): M) \cap((0): I)=(0): M$,

In (5.7) we consider the rings $R_{n}=F[X] /\left(X^{n}\right)$ and use (5.4) to show that $u R_{n}[u, t I]$ is irreducible and $\mathbf{F}\left(R_{n}, I\right)$ is Gorenstein if and only if $I=X^{i}$ with $i$ a divisor of $n$.

(5.7) REMARK. Let $n$ be a positive integer, let $F$ be a field, let $X$ be an indeterminate, let $R_{n}=F[X] /\left(X^{n}\right)=F[x]$, where $x^{n}=0$, and let $M_{n}=x R_{n}$. Then $R_{n}$ is an Artinian Gorenstein local ring and:

(5.7.1) For each positive integer $n$ it is true that $u R_{n}\left[u, t M_{n}\right]$ is irreducible and the form ring $\mathbf{F}\left(R_{n}, M_{n}\right)$ of $R_{n}$ with respect to its maximal ideal $M_{n}$ is Gorenstein.

(5.7.2) For each even positive integer $n$ it is true that $u R_{n}\left[u, t M_{n}^{2}\right]$ is irreducible and the form ring $\mathbf{F}\left(R_{n}, M_{n}^{2}\right)$ of $R_{n}$ with respect to the ideal $M_{n}^{2}$ is Gorenstein.

(5.7.3) For each odd positive integer $n \geq 3, u R_{n}\left[u, t M_{n}^{2}\right]$ is reducible and the form ring $\mathbf{F}\left(R_{n}, M_{n}^{2}\right)$ of $R_{n}$ with respect to the ideal $M_{n}^{2}$ is not Gorenstein.

(5.7.4) More generally, if $i$ is an integer with $1 \leq i \leq n$ and $I=M_{n}^{i}=x^{i} R_{n}$, then $u R_{n}\left[u, t M_{n}^{i}\right]$ is irreducible and the form $\operatorname{ring} \mathbf{F}\left(R_{n}, M_{n}^{i}\right)$ of $R_{n}$ with respect to the ideal $M_{n}^{i}$ is Gorenstein if and only if $n$ is a multiple of $i$.

Proof. It is clear that $R$ is an Artinian Gorenstein local ring, so it suffices to prove (5.7.4), and for this we consider the two cases: (a) $n$ is a multiple of $i$; and, (b) $n$ is not a multiple of $i$.

For (a), let $n=q i$, where $q$ is a positive integer. Then for $j=0,1, \ldots, q-2$ it is readily checked that $T_{j}=\left(x^{i}\right)^{j+1} R_{n}$, that $T_{q-1}=x^{i q-1} R_{n} \supset(0)=\left(x^{i}\right)^{q} R_{n}$, and that $T_{j}=(0)=\left(x^{i}\right)^{j+1} R_{n}$ for $j \geq q$. Therefore (5.4.2) holds, so it follows from (5.4) that $u R_{n}\left[u, t M_{n}^{i}\right]$ is irreducible and that $\mathbf{F}\left(R_{n}, M_{n}^{i}\right)$ is Gorenstein.

For (b), let $n=q i+r$, where $q$ is a nonnegative integer and $1 \leq r<i$. Then it is readily checked that $T_{q-1}=x^{i q-i+r} R_{n} \supset x^{i q} R_{n}$ and that $T_{q}=x^{n-1} R_{n} \supset\left(x^{i}\right)^{q+1} R_{n}$ 
$=(0)$. Therefore (5.4.2) does not hold, so it follows from (5.4) that $u R_{n}\left[u, t M_{n}^{i}\right]$ is not irreducible and that $\mathbf{F}\left(R_{n}, M_{n}^{i}\right)$ is not Gorenstein,

We close this section with an example of an Artinian Gorenstein local ring $(R, M)$ such that $u R[u, t M]$ is reducible and $\mathbf{F}(R, M)$ is not Gorenstein.

(5.8) EXAMPLE. Let $F$ be a field, let $X$ be an indeterminate, let $R=F\left[X^{2}, X^{3}\right] /\left(X^{5}\right)=$ $F\left[x^{2}, x^{3}\right]$, where $x^{5}=0$ and $x^{n}=0$ for $n \geq 7$, and let $M=\left(x^{2}, x^{3}\right) R$. Then $R$ is an Artinian Gorenstein local ring such that $u R[u, t M]$ is reducible and the form ring $\mathbf{F}(R, M)$ of $R$ with respect to its maximal ideal $M$ is not Gorenstein.

Proof. It is clear that $R$ is an Artinian Gorenstein local ring, so by (5.4) it suffices to show that (5.4.2) does not hold. And for this, it is readily checked that $T_{1}=x^{3} R$ $\supset x^{4}=M^{2}$ and that $T_{3}=x^{6} \supset(0)=M^{4}$, so (5.4.2) does not hold,

6. SOME EXAMPLES. In this section we give several examples of the "bad" behavior of the irreducible components of an ideal, even in regular local rings of altitude two. Our first example, (6.2), shows that $\mathbf{S}$ (see (2.1.5)) is not empty, and to prove (6.2.3) we need the following result.

(6.1) PROPOSITION. Let $q$ be an irreducible $M$-primary ideal in a local ring $(R, M)$, let $q_{1}=q: M$ be the unique cover of $q$, and let $(\bar{R}, \bar{M})=(R / q, M / q)$. Then $q_{1}$ has an irreducible cover if and only if $\bar{M}$ covers a principal ideal.

Proof. Let $Q$ be a cover of $q_{1}$. Then since $q_{1}$ is the unique cover of $q$, and since the operation $\bar{I} \rightarrow \bar{I}^{\prime}=(0): \bar{I}$ on the set of ideals $\bar{I}$ of $\bar{R}$ is one-to-one and reverses inclusion (see [ZS, pp. 247-251]), it follows that ${\overline{q_{1}}}^{\prime}=\bar{M}$ is a cover of $\bar{Q}^{\prime}$. Also, $Q$ is irreducible if and only if $\bar{Q}^{\prime}$ is a principal ideal, by [ZS, Theorem 35, p. 250], and the conclusion readily follows from this,

The following example was discussed following (2.5).

(6.2) EXAMPLE. Let $(R, M=(x, y) R)$ be a regular local ring of altitude two and let $n>1$ and $m>1$ be integers. 
(6.2.1) Let $I=\left(x^{n}, x y, y^{m}\right) R$. Then $q_{1}=\left(x^{n}, y\right) R, q_{2}=\left(x, y^{m}\right) R$, and $q_{3}=$ $\left(x y, x^{n-1}+y^{m-1}\right) R$ are in $\operatorname{irr}(I), I=q_{1} \cap q_{2}($ so $n(I)=2), \ell\left(q_{1} / I\right)=m-1, \ell\left(q_{2} / I\right)$ $=n-1$, and $n_{i r r}(I)=1=\ell\left(q_{3} / I\right)$ (so $q_{3}$ is an irreducible cover of $I$ ), so $I \notin \mathbf{S}$ by $(2.5 .2)$.

(6.2.2) If $m=2$ and $I=\left(x^{n}, x^{n-1} y, y^{2}\right) R$, then $q_{1}=\left(x^{n-1}, y^{2}\right) R$ is an irreducible cover of $I$, so $n(I)=2$ and $\ell\left(q_{1} / I\right)=1=n_{\text {irr }}(I)$, so $I \notin \mathbf{S}$ by (2.5.2). (Similarly, if $n=2$ and $I=\left(x^{2}, x y^{m-1}, y^{m}\right) R$, then $q_{2}=\left(x^{2}, y^{m-1}\right) R$ is an irreducible cover of $I$, so $n(I)=2$ and $\ell\left(q_{2} / I\right)=1=n_{\text {irr }}(I)$, so $I \notin \mathbf{S}$ by (2.5.2).)

(6.2.3) Let $n>2, m>2$, and $I=\left(x^{n}, x^{n-1} y^{m-1}, y^{m}\right) R$. Then $q_{1}=\left(x^{n}, y^{m-1}\right) R$ and $q_{2}=\left(x^{n-1}, y^{m}\right) R$ are in $\operatorname{irr}(I), I=q_{1} \cap q_{2}$ (so $\left.n(I)=2\right), \ell\left(q_{1} / I\right)=m-1$, $\ell\left(q_{2} / I\right)=n-1$, and $I$ has no irreducible cover, so $I \in \mathbf{S}$.

Proof. For (6.2.1), it is shown in [HRS4, (2.1.2), (3.1) and (4.1)] that $q_{1}$ and $q_{2}$ are in $\operatorname{irr}(I)$ and that $I=q_{1} \cap q_{2}$. Also, it is readily checked that $I \subset\left(x^{n-1}, x y, y^{m}\right) R \subset$ $\cdots \subset\left(x^{2}, x y, y^{m}\right) R \subset\left(x, y^{m}\right) R=q_{2}$ is a saturated chain of ideals between $I$ and $q_{2}$ (so $\left.l\left(q_{2} / I\right)=n-1\right)$ and that $I \subset\left(x^{n}, x y, y^{m-1}\right) R \subset \cdots \subset\left(x^{n}, x y, y^{2}\right) R \subset\left(x^{n}, y\right) R=$ $q_{1}$ is a saturated chain of ideals between $I$ and $q_{1}$ (so $\left.l\left(q_{1} / I\right)=m-1\right)$. Further, it is readily checked that $q_{3}$ is a cover of $I\left(\right.$ so $\left.l\left(q_{3} / I\right)=1\right)$, and $q_{3}$ is irreducible (since it is generated by a system of parameters), so $q_{3} \in \operatorname{irr}(I)$ by (2.5.2). It therefore follows that $I \notin \mathbf{S}$.

For (6.2.2), it is readily checked that $q_{1}$ is an irreducible cover of $I$, and the conclusions readily follow from this.

Finally, for (6.2.3), it is shown in [HRS4, (2.1.2), (3.1) and (4.1)] that $q_{1}$ and $q_{2}$ are in $\operatorname{irr}(I)$ and that $I=q_{1} \cap q_{2}$. Also, it is readily checked that $I \subset\left(x^{n}, x^{n-2} y^{m-1}, y^{m}\right) R \subset$ $\cdots \subset\left(x^{n}, x y^{m-1}, y^{m}\right) R \subset\left(x^{n}, y^{m-1}\right) R=q_{1}$ is a saturated chain of ideals between $I$ and $q_{1}\left(\right.$ so $\left.l\left(q_{1} / I\right)=n-1\right)$, and a similar chain shows $l\left(q_{2} / I\right)=m-1$. Finally, it follows from (6.1) (with $q=\left(x^{n}, y^{m}\right) R$ and $q_{1}=I$ ) that if $n>2$ and $m>2$, then $I$ has no irreducible cover (since if $\bar{M}=(\bar{x}, \bar{y}) \bar{R}$ covers $\overline{b R}$, then $\bar{M}=(\bar{b}, \bar{c}) \bar{R}$ (for some $c \in M)$ and $\bar{c} \bar{M} \subseteq \overline{b R}$, so $\bar{M}^{2} \subseteq \overline{b R}$, hence $\left(x^{2}, x y, y^{2}\right) R \subseteq\left(x^{n}, y^{m}, b\right) R$, and this clearly cannot happen if $n>2$ and $m>2$ ),

The examples in (6.2) show some of the things that do not necessarily hold in a 
given irreducible decomposition of an $M$-primary ideal $I$, as noted in the following remark.

(6.3) REMARK. Let $I=q_{1} \cap \cdots \cap q_{g}$ be an irredundant irreducible decomposition of $I$. Then:

(6.3.1) (6.2.1) shows that it is possible that $\ell\left(q_{i} / I\right)>n_{\text {irr }}(I)$ for $i=1, \ldots, g$.

(6.3.2) (6.2.1) shows that it is possible (by varying $I$ ) that, for $i=1, \ldots, g$, there is no bound on $\ell\left(q_{i} / I\right)$, even when $n_{i r r}(I)=2$.

The next two examples were rather a surprise to us. The first of these shows that, even if $n(I)=2$, there may exist arbitrarily long finite chains of ideals in $\mathbf{I}(I)$ each of which is the intersection of two ideals in $\operatorname{irr}(I)$, and the second shows that there may exist ideals in $\mathbf{I}(I)$ that are the intersection of more than $n(I)$ elements in $\operatorname{irr}(I)$.

(6.4) PROPOSITION. Let $(R, M)$ be a regular local ring of altitude two. Then there exists an infinite chain of $M$-primary ideals $I_{1} \supset I_{2} \supset \cdots$ and an infinite set $\mathbf{Q}$ of irreducible $M$-primary ideals $q_{n}$ such that for all positive integers $n$ and $k$ it holds that $I_{n+k}$ is the irredundant intersection $q_{n} \cap q_{n+k}$. In particular, for each positive integer $n$ the ideals in $\mathbf{Q}$ that are in $\operatorname{irr}\left(I_{n}\right)$ are the ideals $q_{1}, \ldots, q_{n}$.

Proof. Fix $x_{1} \in M-M^{2}$, let $q_{1}=\left(x_{1}, M^{2}\right) R$, and let $I_{1}=\left(x_{1} M, M^{2}\right) R$, so $I_{1}=$ $M^{2}$. Then:

$\left(a_{1}\right) M^{2} \subseteq I_{1} \subseteq q_{1}$ and $M \nsubseteq q_{1} ;$ and,

$\left(b_{1}\right) q_{1}=\left(x_{1}, I_{1}\right) R$ is a cover of $I_{1}$

(since $x_{1} M \subset M^{2}=I_{1}$ and $q_{1}=I_{1}+x_{1} R$ ). (Therefore if $y \in M-x_{1} R$ and if we let $q=\left(y, I_{1}\right) R$, then it is readily checked that $q_{1} \cap q=I_{1}$, so $q_{1}$ and $q$ are in $\operatorname{irr}\left(I_{1}\right)$.)

Now let $z_{1} \in M-q_{1}$, let $x_{2}=z_{1}+x_{1}$, let $q_{2}=\left(x_{2}, M^{3}\right) R$, and let $I_{2}=$ $\left(x_{2} M, M^{3}\right) R$. Then:

$\left(a_{2}\right) M^{3} \subseteq I_{2} \subseteq q_{2}$ and $M^{2} \nsubseteq q_{2} ;$ and,

$\left(b_{2}\right) q_{2}=\left(x_{2}, I_{2}\right) R$ is a cover of $I_{2}$. 
Therefore assume that $n \geq 2$ and that $z_{n-1} \in M^{n-1}-q_{n-1}, x_{n}=z_{n-1}+x_{n-1}$, $q_{n}=\left(x_{n}, M^{n+1}\right) R$, and $I_{n}=\left(x_{n} M, M^{n+1}\right) R$ have been defined so that:

$\left(a_{n}\right) M^{n+1} \subseteq I_{n} \subseteq q_{n}$ and $M^{n} \nsubseteq q_{n} ;$ and,

$\left(b_{n}\right) q_{n}=\left(x_{n}, I_{n}\right) R$ is a cover of $I_{n}$.

Then let $z_{n} \in M^{n}-q_{n}, x_{n+1}=z_{n}+x_{n}, q_{n+1}=\left(x_{n+1}, M^{n+2}\right) R$, and $I_{n+1}=$ $\left(x_{n+1} M, M^{n+2}\right) R$.

Then it is readily checked that:

$\left(a_{n+1}\right) M^{n+2} \subseteq I_{n+1} \subseteq q_{n+1}$ and $M^{n+1} \nsubseteq q_{n+1} ;$ and

$\left(b_{n+1}\right) q_{n+1}=\left(x_{n+1}, I_{n+1}\right) R$ is a cover of $I_{n+1}$.

Also, for each $n$ it follows that $x_{n} \in M-M^{2}$. (For, $x_{1} \in M-M^{2} ; x_{2}=x_{1}+z_{1}$ and $z_{1} \in M-x_{1} R$, so $x_{2} \in M-M^{2}$; and, if $i>2$ and $x_{i} \in M-M^{2}$, then $z_{i}$ $\in M^{i}-q_{i}$ implies that $x_{i+1}=x_{i}+z_{i} \in M-M^{2}$.) Therefore $q_{n}=\left(x_{n}, M^{n+1}\right) R$ is generated by a system of parameters (since $R / x_{n} R$ is a PID), hence each $q_{n}$ is irreducible.

Further, for each $n$ it follows that $I_{n+1} \subset I_{n}$. (For $I_{n+1}=\left(x_{n+1} M, M^{n+2}\right) R$ and $x_{n+1} M=\left(x_{n}+z_{n}\right) M \subseteq x_{n} M+z_{n} M \subseteq x_{n} M+M^{n} M=I_{n}$, and $\left(a_{n}\right)$ and $\left(a_{n+1}\right)$ show that the containment is proper.)

Moreover, $\left(a_{n}\right)$ and $\left(a_{n+k}\right)$ show that $q_{n} \nsubseteq q_{n+k}$ (since $M^{n+1} \subseteq q_{n}$ and $M^{n+k} \nsubseteq$ $\left.q_{n+k}\right)$. Therefore, if it is shown that, for each $n$ and $k, q_{n+k} \nsubseteq q_{n}$, then it follows from $\left(b_{n+k}\right)$ that $q_{n} \cap q_{n+k}=I_{n+k}$ is an irredundant intersection. And it then follows that $q_{1}, \ldots, q_{n}$ are in $\operatorname{irr}\left(I_{n}\right)$, and $q_{n+i} \notin \operatorname{irr}\left(I_{n}\right)$ for all $i \geq 1$, since $q_{n+i}$ is a cover of $I_{n+i}$ and $I_{n+i}$ is properly contained in $I_{n}$ (so $I_{n} \nsubseteq q_{n+i}$ ). Therefore it remains to show that $q_{n+k}$ is not contained in $q_{n}$.

For this, suppose that $q_{n+k} \subseteq q_{n}$. Then it follows from the definition of the ideals $q_{i}$ that $x_{n+k} \in q_{n}=\left(x_{n}, M^{n+1}\right) R$, so there exist $r \in R$ and $m \in M^{n+1}$ such that $x_{n+k}=r x_{n}+m$. Also, the definition of the $x_{i}$ shows that $x_{n+k}=$ $x_{n}+z_{n}+z_{n+1}+\cdots+z_{n+k}$, and the definition of the $z_{i}$ shows that $z_{n} \notin M^{n+1}$ and that $z_{n+i} \in M^{n+1}$ for $i=1, \ldots, k$. Therefore $x_{n+k}=x_{n}+z_{n}+m^{\prime}$, where $m^{\prime}=$ $z_{n+1}+\cdots+z_{n+k} \in M^{n+1}$. Therefore it follows that $r x_{n}+m=x_{n+k}=x_{n}+z_{n}+m^{\prime}$, so $z_{n}=(r-1) x_{n}+m-m^{\prime} \in\left(x_{n}, M^{n+1}\right) R=q_{n}$, and this contradicts the choice of 
$z_{n} \in M^{n}-q_{n}$. Therefore $q_{n+k} \nsubseteq q_{n}$ for all $n$ and $k$,

Concerning the set $\mathbf{Q}$ in (6.4), note that the intersection of each set of more that two elements in $\mathbf{Q}$ is redundant. From this observation, a natural question is, if $n(I)=k$, then is the intersection of each set of $h>k$ elements in $\operatorname{irr}(I)$ redundant? The answer is no, as the next result shows.

(6.5) PROPOSITION. Let $(R, M)$ be a regular local ring of altitude two and let $k<m$ be positive integers. Then there exists an open ideal $I$ of $R$ such that $n(I)=$ $m$ and there exist $m+k$ elements in $\operatorname{irr}(I)$ whose intersection is irredundant.

Proof. It is shown in the proof of [HS, (2.1)] by computing $\operatorname{Tor}(R / I, R / M)$ in two ways via projective resolutions of $R / I$ and $R / M$ that if $I$ is an open ideal in $R$, then $n(I)=v(I)-1$, where $v(I)$ denotes the number of elements in a minimal basis of $I .{ }^{3}$ Also, given positive integers $k<m$, [HRS4, (3.12)] shows that in every regular local ring of altitude two there exists an open ideal $I$ such that $v(I)=m+1$ and $v(I: M)=m+k+2$. Therefore there exists an ideal $J$ in $R$ such that $I \subset J \subset$ $I: M$ and $v(J)=m+k+1$. Then it follows that $n(I)=m$ and $n(J)=m+k$, and $J$ is the (irredundant) intersection of $m+k$ elements in $\operatorname{irr}(I)$, by (3.2),

A specific example of ideals $I$ and $J$ such that $I \subset J \subset I: M$ with $n(I)=m$ and $n(J)=m+k$ as in (6.5) is the following: let $n=m+1, s=k+2$, for $i=$ $1, \ldots, s$ let $f_{i}=x^{2(i-1)} y^{n+s-2 i}$ and $z_{i}=x^{2 i-1} y^{n+s-2 i-1}$, for $i=s+1, \ldots, n$ let

$f_{i}=x^{s+(i-1)} y^{n-i}$ and $z_{i}=x^{s+(i-1)} y^{n-1-i}$ (so $f_{i} \in z_{i-1} R$ for $i=s+1, \ldots, n ; z_{n}$ is not used), and, finally, let $I=\left(f_{1}, \ldots, f_{n}\right) R$ and $J=\left(f_{1}, \ldots, f_{s}, z_{2}, \ldots, z_{n-1}\right) R$. Then the proof of [HRS4, (3.11)] shows that $I \subset J \subset I: M, v(I)=n(=m+1)$, and $v(J)=s+n-2(=m+k+1)$, so $n(I)=m$ and $n(J)=m+k$ (as noted at the start of the proof of (6.5)).

\section{ACKNOWLEDGMENT}

We thank Craig Huneke for sharing with us his insight on Theorem 4.1.

\footnotetext{
${ }^{3}$ See Section 3 of [HRS1] for other applications of this result.
} 


\section{REFERENCES}

[BH] W. Bruns and J. Herzog, Cohen-Macaulay Rings, Cambridge University Press, Cambridge, 1993.

[BS] D. Bayer and M. Stillman, MACAULAY, Computer Algebra System.

[Gi] R. Gilmer, Chapter 8 "Commutative Ring Theory" of Emmy Noether A Tribute to Her Life and Work, Marcel Dekker, New York, 1981.

[Gr] W. Gröbner, Über irreducible Ideale in kommutativen Ringen, Math. Ann. 110 (1934), 197-222.

[HRS1] W. Heinzer, L. J. Ratliff, Jr., and K. Shah, On the embedded primary components of ideals, II, J. Pure Appl. Algebra 101 (1995), 139-156.

[HRS2] W. Heinzer, L. J. Ratliff, Jr., and K. Shah, On the embedded primary components of ideals, III, J. Algebra 171 (1995), 272-293.

[HRS3] W. Heinzer, L. J. Ratliff, Jr., and K. Shah, On the embedded primary components of ideals, $I V$, Trans. Aner. Math. Soc. 347 (1995), 701-708.

[HRS4] W. Heinzer, L. J. Ratliff, Jr., and K. Shah, Parametric decompositions of monomial ideals, I, Houston J. Math. 21 (1995), 29-52.

[HR] M. Hochster and L. J. Ratliff, Jr., Five theorems on Macaulay rings, Pacific J. Math. 44 (1973), 147-172.

[HS] C. Huneke and J. Sally, Birational extensions in dimension two and integrally closed ideals, J. Algebra 115 (1988), 481-500.

[M] H. Matsumura, Commutative Algebra, Second Edition, Benjamin/Cummings, Advanced Book Program, Reading, Massachusetts, 1980.

[N] M. Nagata, Local Rings, Interscience Tracts in Pure and Applied Math., No. 13, Interscience, New York, NY, 1962.

[No] E. Noether, Idealtheorie in Ringbereichen, Math. Ann 83 (1921), 24-66.

[RR] L. J. Ratliff, Jr. and D. Rush, Notes on ideal covers and associated primes, Pacific J. Math. 73 (1977), 169-191.

[SV] D. W. Sharpe and P. Vamos, Injective Modules, Cambridge Univ. Press, London/New York, 1972.

[ZS] O. Zariski and P. Samuel, Commutative Algebra, Vol. 1, D. Van Nostrand Co., Inc., Princeton, 1958. 